Cite this: Phys. Chem. Chem. Phys., 2011, 13, 5430-5440

\title{
Ion current rectification and rectification inversion in conical nanopores: a perm-selective view $\dagger \ddagger$
}

\author{
Dmitry Momotenko, ${ }^{a}$ Fernando Cortés-Salazar, ${ }^{a}$ Jacques Josserand, ${ }^{a}$ Shujuan Liu, ${ }^{b}$ \\ Yuanhua Shao ${ }^{b}$ and Hubert H. Girault* $* a$
}

Received 19th November 2010, Accepted 11th February 2011

DOI: $10.1039 / \mathbf{c 0 c p 0 2 5 9 5 j}$

Ionic transport in charged conical nanopores is known to give rise to ion current rectification. The present study shows that the rectification direction can be inverted when using electrolyte solutions at very low ionic strengths. To elucidate these phenomena, electroneutral conical nanopores containing a perm-selective region at the tip have been investigated and shown to behave like classical charged nanopores. An analytical model is proposed to account for these rectification processes.

\section{Introduction}

Recently, a lot of attention has been paid to the transport properties of electrolytes in nanoscale pores and tubes. Apart from the direct biological applications of ion transport through biological membranes, ${ }^{1}$ nanopores and nanochannels have found applications in a variety of separation techniques, ${ }^{2,3}$ molecule delivery devices, ${ }^{4}$ sensing systems, ${ }^{5,6}$ and have been integrated within microfluidic chips. ${ }^{7}$ Additionally, nanopipettes have been applied in bionanotechnology, ${ }^{8}$ living cells studies, ${ }^{9}$ as probes for scanning ion conductance microscopy $(\mathrm{SICM})^{10,11}$ for high-resolution imaging of surface topography and to study the kinetics of ion transfer reactions at liquidliquid interfaces supported at the tip of nanopipettes. ${ }^{12}$ The ion transport properties in such nanosystems and their different applications are strongly governed by surface charge effects and surface chemistry due to the high surface to volume ratio in these nanostructures.

It was found that the transport of charged species within asymmetric cone-shaped nanopores and nanopipettes exhibited specific behavior, called ion current rectification (ICR). ${ }^{13}$ ICR is encountered when the magnitude of the current through a nanopore depends on the polarity of the applied biases, leading

\footnotetext{
${ }^{a}$ Laboratoire d'Electrochimie Physique et Analytique,

Ecole Polytechnique Fédérale de Lausanne, Station 6,

CH-1015 Lausanne, Switzerland.E-mail:hubert.girault@epfl.ch;

Fax: + 41-21-693 3667; Tel: + 41-21-693 3145

${ }^{b}$ College of Chemistry and Molecular Engineering, Peking University, Beijing 100871, China

$\dagger$ This article is part of the special collection on Interfacial processes and mechanisms in celebration of John Albery's 75th birthday.

$\ddagger$ Electronic supplementary information (ESI) available: Details of the validation procedure for FEM simulations; electroneutrality maps at the tip of a charged nanopore; relative ionic conductivity distributions within the pore at very low electrolyte concentrations; current-voltage characteristics of uncharged pores presenting permselectivity at the tip. See DOI: $10.1039 / \mathrm{c} 0 \mathrm{cp} 02595 \mathrm{j}$
}

to a non-ohmic current-voltage behavior. Such asymmetric behavior can be observed if (a) the opening diameter of the nanopore tip is comparable to the thickness of the electrical diffuse layer (EDL) also called the Gouy-Chapman layer, (b) the wall carries an excess surface charge, and (c) asymmetric interaction between charged walls and ions in solution occurs. ${ }^{14}$ The latter condition can be fulfilled if the geometry of the pore is non-symmetric, if there is asymmetry of the surface charge density within the pore or if some electrolyte concentration difference inside the pore takes place. Thereby, ion current rectification phenomena could be found in charged conical nanopores, ${ }^{14}$ nanochannels and pores with charge density gradients, ${ }^{15}$ wall charge asymmetry ${ }^{16}$ or in homogenous nanochannels with asymmetric electrolyte concentrations. ${ }^{17}$

A few mechanisms have been proposed to explain ICR. It has been suggested that ICR could arise due to the changes of the size of pore's opening, caused by the applied voltage. ${ }^{18}$ Although such situation could be realized experimentally ${ }^{19}$ (with the use of an electromechanical gate, formed by DNA oligomers, chemisorbed on the pore walls and surface of the membrane), the most common explanation of rectification phenomena for more conventional systems assumes interactions between electrolyte ions and fixed charged groups of the walls.

As it was proposed in a phenomenological report of Wei et al. ${ }^{13}$ such interactions lead to the existence of a small perm-selective region at the tip. The model considers this region as the link between two conical zones where ionic transport occurs inside and outside the pore. The analytical expression for ionic fluxes shows the origin of rectification as the asymmetry of conical regions inside and outside the pore coupled to the perm-selectivity at the tip.

Siwy and co-workers ${ }^{20}$ proposed that rectification originates from a ratchet mechanism based on ion trapping by the electric potential well formed near the tip of the pore. Another 
mechanism developed by Woermann ${ }^{21,22}$ specifies that the pore could be divided into three regions according to the electrolyte concentration, and high- and low-conductance states, which causes the difference in ionic currents for positive and negative potentials, are the consequence of increasing and decreasing ionic strength within the pore. According to this mechanism, ICR is a result of a high selectivity of the pore to the counter-ions of the EDL. High electric conductance states, with higher current values, occur when a region of high ionic strength is formed inside the pore. Lower current values are caused by a decrease of ionic strength within the nanopore, which leads to a decrease of ionic conductivity and consequently lower conductance of the nanopore.

Computations made by Cervera et al. $^{23}$ and White ${ }^{24}$ have confirmed the hypothesis that this non-ohmic current-voltage behavior is related to the conductivity value inside the pore, which is dependent on the ionic strength within the pore and especially in the tip region. Thus, ICR should be strongly governed by the tip diameter and the pore shape. ${ }^{25,26}$

An electroconvection mechanism was also proposed to explain the diode-like behavior in porous membranes. ${ }^{27}$ It is based on the suggestion that high-conducting states exist due to convectional mixing, which leads to an increase in the electrical current passing through the membrane. Such nongravitational convection ("electroconvection" or "electro-osmosis of the second kind") probably leads to mixing of the depletion layer within the membrane with the adjacent region of the bulk phase. The conclusion is that this process would be especially effective in an electrolyte-depleted zone. However the work was not continued and no experimental proofs were found.

In this manuscript, we report the results of numerical simulations of the ICR phenomenon in nanopores of conical geometry using the finite element method (FEM). This paper emphasizes the basic aspects of the ICR mechanism, i.e. the origin of current asymmetry and the reasons for rectification inversion. The observed behavior is explained with the use of analytical and numerical models, describing perm-selective pores. The results are validated by comparison with analytical expressions, when possible, and with other literature data reported.

\section{Theory and numerical description}

\section{Basic equations}

The ionic transport in electrolyte solutions can be described with the Nernst-Planck equation (eqn (1))

$$
J_{i}=-D_{i} \nabla c_{i}-\frac{z_{i} F}{R T} D_{i} c_{i} \nabla \phi+c_{i} v
$$

The total flux $J_{i}$ of species $i$ is described as a sum of mass transport, caused by diffusion $-D_{i} \nabla c_{i}$, migration $-z_{i} F D_{i} c_{i}(R T)^{-1} \nabla \phi$ and convection $c_{i} v$. Here $D_{i}, c_{i}$ and $z_{i}$ specify diffusion coefficient, concentration and charge number of species $i$, respectively. $F, R, T, v$ and $\phi$ are the Faraday constant, the gas constant, temperature, fluid velocity and the Galvani electric potential, respectively.
The electrostatic interactions between charged species of the electrolyte solution and a charged wall are determined by the Poisson equation (eqn (2))

$$
\nabla^{2} \phi=-\frac{F}{\varepsilon \varepsilon_{0}} \sum_{i} z_{i} c_{i}
$$

where $\varepsilon$ and $\varepsilon_{0}$ denote the relative permittivity of the medium and the vacuum permittivity, respectively.

The general mass transport equation can be simplified in the case of absence of convection, so that last term of eqn (1) can be ignored, and by assuming a steady-state regime $\left(\nabla \cdot J_{i}=0\right)$. The electro-osmotic flow (EOF), which can contribute to the flux, is neglected in this model since the relative value of EOF is small for the considered system. Such assumption can be applied for systems with relatively small surface charge density and low electric field within the pore, as the contribution of the electro-osmotic flow to ICR increases with increasing wall charge and applied biases. ${ }^{24}$

By coupling equations eqn (1) and (2) and solving the system with appropriate boundary conditions the spatial distributions of the Galvani potential $\phi$ and the ionic concentrations $c_{i}$ can be obtained. Given the complexity of conical nanopore systems, numerical solutions, e.g. finite-difference or finite-element methods are often used.

\section{FEM simulations procedure}

The FEM simulations were performed using the finite element software COMSOL Multiphysics (version 3.5a), operated on Linux Ubuntu 8.04 platform with a four Core Mac Pro $2.66 \mathrm{GHz}$ CPU and $9.8 \mathrm{~GB}$ of RAM. The Nernst-Planck equations without electroneutrality and the Poisson equation were solved in dimensional form for the given two-dimensional axisymmetric geometries of a nanopore (Fig. 1a). Only half of the cylindrical model was simulated. The computational domain presented in Fig. 1a is the conically shaped axisymmetric channel of $5 \mu \mathrm{m}$ length, placed between two equal reservoirs of solution. Such configuration allows the simulations of realistic conditions of mass transport at the entrance and the exit of the nanopore. The size of the reservoirs and the length of the charged region outside the pore were adapted to have no influence on the results. The simulated nanopore is characterized by the radius of the tip, which was fixed to the value of $10 \mathrm{~nm}$, and the half-cone angle $\alpha$ was set to $30.87^{\circ}$. The cone base radius was varied for obtaining the proper nanopore's semi-angle in order to study the effect of the pore geometry on the value of ICR.

The numerical solutions of eqn (1) and (2) were performed with the boundary conditions presented in Table 1 .

In the present work, we investigate the ICR phenomenon for aqueous solutions of $\mathrm{KCl}$ (with relative permittivity $\varepsilon=80$ ), at different bulk concentrations $c_{0}$. The chosen diffusion coefficients $D_{i}$ of the ions were $2.0 \times 10^{-9} \mathrm{~m}^{2} \mathrm{~s}^{-1}$ for both $\mathrm{K}^{+}$and $\mathrm{Cl}^{-}$. The nanopore carries the charge density $\sigma=-1 \mathrm{mC} \mathrm{m}^{-2}$. This value can be attributed to the surface charge density of the glass or silica surfaces in contact with highly diluted aqueous electrolyte solutions. However, the wall charge depends also on the $\mathrm{pH}$, the number of active surface sites and the $\mathrm{p} K_{\mathrm{a}}$ of the corresponding dissociation reaction. ${ }^{28}$ 

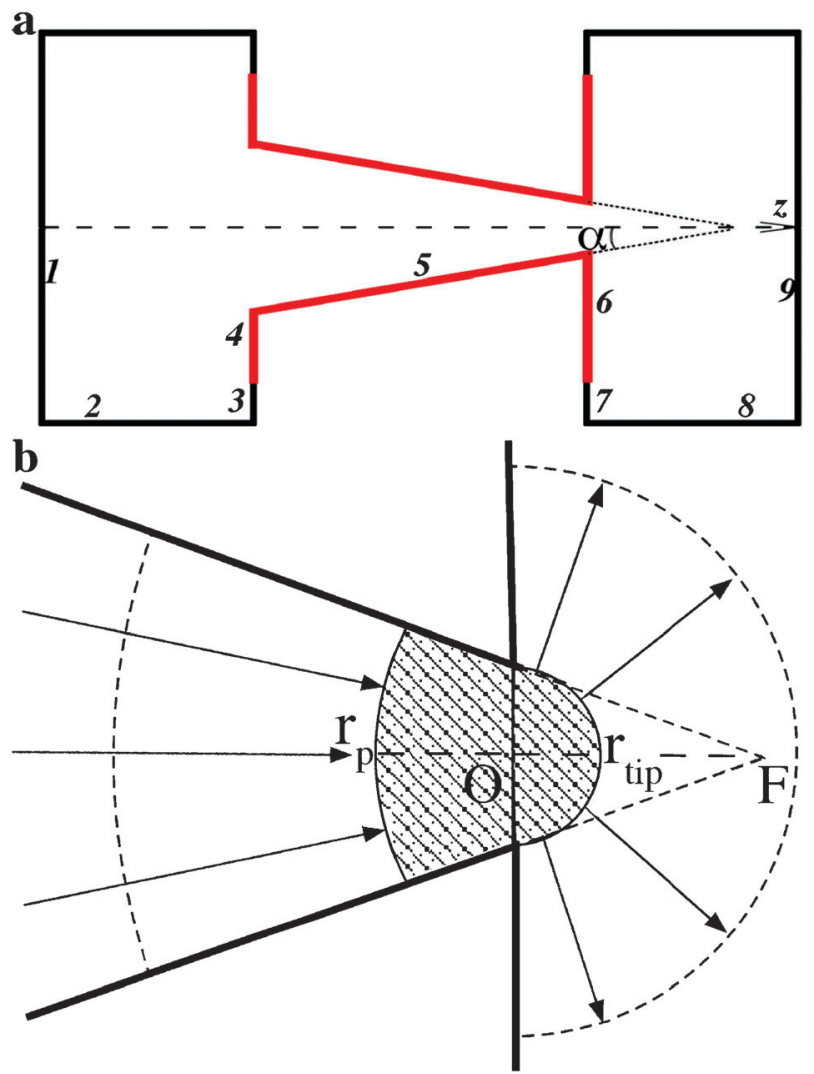

Fig. 1 (a) Schematic representation of the computational domain (not in a scale) of the conical nanopore, placed between two equal reservoirs of solution. The numbers specify the surfaces with appropriate boundary conditions, shown in Table 1 . The presence of a wall charge is marked with red. Note that only half of the pore, symmetric along the $z$ axis (dashed line) is simulated. Dotted lines specify the conical shape of the nanopore and $\alpha$ indicates the half-cone angle. (b) An illustration of ionic transport in a non-charged pore. Surfaces of a truncated sphere (inside the pore) and a hemisphere (outside) are indicated with dashed and solid lines and centered at points $\mathrm{F}$ and $\mathrm{O}$, respectively. The dash-dotted domain illustrates the perm-selective plug at the tip and the arrows specify ionic fluxes.

The numerical solution of the coupled Nernst-Planck and Poisson equations was computed, assuming room-temperature conditions. The mesh was refined down to a value of $0.5 \mathrm{~nm}$ at the charged wall regions to resolve the features of a diffuse double layer. The bias is applied as a potential difference between two equipotentials, specified as surfaces 1 and 9 in Fig. 1a, i.e. $\Delta \phi=\phi_{1}-\phi_{9}$.
The simulations of the perm-selective nanopore assume absence of wall charges, but presence of a thin $(0.5-100 \mathrm{~nm})$ region, where anions cannot permeate. The Nernst-Planck equation describing an anion flux in the perm-selective subdomain was excluded from the computations, as well as the Poisson equation. Consequently, only the cation flux described by the Nernst-Planck equation without electroneutrality was allowed in the perm-selective zone.

The details of the validation procedure are given in the ESI (see Fig. S-1).†

\section{Results and discussion}

\section{Resistance distribution for an uncharged conical nanopore}

Before dealing with the ICR phenomena, it is worth to discuss the resistance distribution at an ideal uncharged nanopore filled with an electrolyte solution, i.e. to consider ohmic aspects. Outside the pore, the equipotential lines can be considered as hemispheres centered on the origin of the $z$-axis (point $\mathrm{O}$ in Fig. 1b). Indeed, by analogy with a microdisc electrode, we can consider radial current distribution. Inside the conical pore that is in fact a truncated sphere defined by a solid angle of semi-angle $\alpha$ (see Fig. 1a), the equipotential lines are also truncated sphere but centered on the "focal" point of the nanopore (point $\mathrm{F}$ in Fig. 1b).

The resistance of the solution outside the pore can therefore be approximated by

$$
R_{o}=\frac{\rho}{2 \pi} \int_{r_{\text {tip }}}^{\infty} \frac{\mathrm{d} r}{r^{2}}=\frac{\rho}{2 \pi r_{\text {tip }}}=k_{\text {out }} \rho
$$

where $\rho$ represents the resistivity of the solution, $r_{\text {tip }}$ the radius of the pore tip and $k_{\text {out }}$ represents the geometric cell constant for the solution phase outside the pore. Similarly, the resistance of the solution inside the pore is given by

$$
R_{i}=\frac{\rho}{\Omega} \int_{r_{\text {tip }} / \sin \alpha}^{\infty} \frac{\mathrm{d} r}{r^{2}}=\frac{\rho}{4 \pi r_{\text {tip }}}\left(\frac{\sin \alpha}{\sin ^{2}\left(\frac{\alpha}{2}\right)}\right)=k_{\text {in }} \rho
$$

with $k_{\text {in }}$ being the geometric cell constant for the solution phase inside the pore, and $\Omega$ the solid angle defining the cone, and given by

$$
\Omega=4 \pi \sin ^{2}\left(\frac{\alpha}{2}\right)=2 \pi(1-\cos \alpha)
$$

Table 1 Boundary conditions for numerical simulations of ICR in conical nanopores

\begin{tabular}{llll}
\hline & & Boundary conditions & \\
\cline { 3 - 4 } Surface & Physical property & Nernst-Planck equations & Poisson equation \\
\hline 1 & Equiconcentration, equipotential & Concentration, $c_{i}=c_{0}$ & Electric potential, $\phi=\phi_{1}$ \\
$2,3,7,8$ & Insulating wall & Insulation, $-\mathbf{n} \cdot J_{i}=0$, & Zero charge, $\mathbf{n} \cdot\left(-\varepsilon_{0} \varepsilon_{r} \nabla \phi\right)=0$ \\
$4-6$ & Glass wall & Insulation, $-\mathbf{n} \cdot J_{i}=0$ & Surface charge, $-\mathbf{n} \cdot\left(-\varepsilon_{0} \varepsilon_{r} \nabla \phi\right)=-\sigma$ \\
9 & Equiconcentration, equipotential & Concentration, $c_{i}=c_{0}$ & Electric potential, $\phi=\phi_{9}$ \\
$z$ axis & Axial symmetry & Axial symmetry & Axial symmetry
\end{tabular}

The numbers specifying the surfaces are correlated to the schematic representation of the nanopore's geometry shown in Fig. 1a (n denotes the vector normal to the surface). 
Therefore the resistance inside the pore is proportional to the one outside and this factor depends on semi-angle $\alpha$ values. For a very small semi-angle of $5^{\circ}$, the resistance for the solution inside the pore is about 23 times larger than outside, but the ratio drops to less than 4 for a semi-angle $\alpha=30.87^{\circ}$ taken as an example for the remainder of this paper. The total resistance for this system therefore simply reads

$$
R_{t}=R_{i}+R_{o}=\frac{\rho}{2 \pi r_{\text {tip }}}\left(1+\cot \frac{\alpha}{2}\right)=k_{\text {tot }} \rho
$$

As shown below, this simple model is reasonably well corroborated by the simulation.

\section{Gouy-Chapman diffuse layer interaction and Donnan potential distribution in the absence of a potential bias}

In the absence of an applied potential difference, the system is at equilibrium and a constant Galvani potential (taken here as equal to $0 \mathrm{~V}$ ) can be observed, except in the space-charged region where a Gouy-Chapman diffuse layer occurs. Usually, the thickness of the diffuse layer, defined by the Debye length, varies with the square root of the electrolyte concentration, ranging from a few to tens of nanometres. When the pore opening is in nanometre size, an interaction between the diffuse layers is possible resulting in the exclusion of anions near negatively charged walls and associated enrichment of cations. Fig. 2 illustrates the influence of electrolyte concentration on the Galvani potential value along the central axis both inside and outside the pore. The overlap of the Gouy-Chapman diffuse layers causes a decrease of the Galvani potential value near the pore tip, in a region where the electroneutrality of the solution is broken. As the electrolyte concentration decreases, the overlap occurs deeper into the pore away from the tip, but also further outside the tip and the Galvani potential decreases accordingly (dashed and doted lines in Fig. 2). At equilibrium, a static electric field along the central axis is established by the

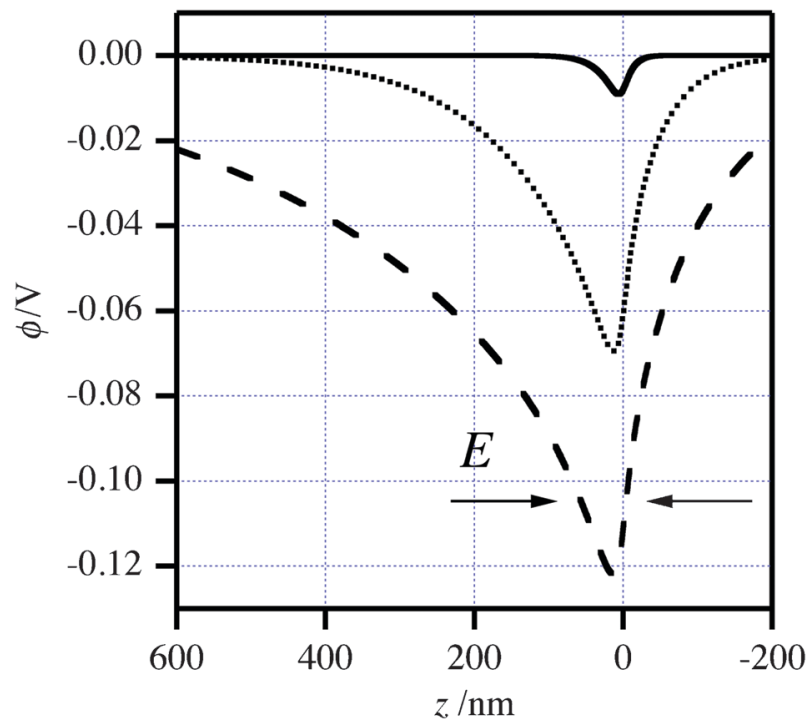

Fig. 2 Electrical potential distributions along the $z$ axis of a negatively charged nanopore in the static case (no bias applied) for different electrolyte concentrations (1, 0.04 and $0.005 \mathrm{mM} \mathrm{KCl}$ for solid, dotted and dashed lines, accordingly). The pore mouth is located at $z=0$. ion distribution, directed towards the tip from both directions, as if concentrating the cations in the tip region. The potential difference observed along the central axis is a Donnan potential difference as classical observed between a perm-selective membrane and an aqueous electrolyte.

\section{Gouy-Chapman diffuse layer interaction and rectification in the presence of a potential bias}

When a bias, i.e. a Galvani potential difference, is applied between the two bulk solutions, the system reaches a steady state with a constant current flowing through the nanopore. At high electrolyte concentrations, the diffuse layers interact only at the very tip, and the Galvani potential distribution along the central axis is monotonous. The electric field direction then only depends on the polarity of the bias. It is interesting to note that in this case the potential distribution is almost not affected in absolute values by the bias polarity as we have:

$$
\phi_{+}(z) \approx-\phi_{-}(z)
$$

where $\phi_{+}(z)$ and $\phi_{-}(z)$ are the potential distribution at positive and negative biases, respectively.

At lower electrolyte concentrations, the overlap of the Gouy Chapman layers takes place deeper into the pore, the Galvani potential profiles present a minimum near the tip of the pore as for the equilibrium case shown in Fig. 3, both at positive and negative biases. As a result, the Galvani potential difference along the $z$-axis still reaches negative values as shown in Fig. $3 a$ and $b$, respectively. The potential minima are almost independent of the bias polarity, being equal to $-0.03 \mathrm{~V}$ for $0.04 \mathrm{mM}$ and $-0.08 \mathrm{~V}$ for $5 \mu \mathrm{M}$, compared to $-0.07 \mathrm{~V}$ and $-0.12 \mathrm{~V}$ for the equilibrium case, respectively. However, the position of the potential minima depends on the polarity of the bias and this is a key issue when discussing rectification inversion (vide infra).

The current density $j$ throughout the system is directly proportional to the electric field $E$

$$
j=\sigma E
$$

where $\sigma$ is the local ionic conductivity given by

$$
\sigma=F \sum_{i} c_{i}\left|z_{i}\right| u_{i}
$$

with $u_{i}$ as the electrophoretic mobility of the species $i$. We shall define a normalized ionic conductivity as the local conductivity normalized by the bulk value. In the case of $\mathrm{KCl}$ solutions for which the two ions have the same electrophoretic mobility, we have simply:

$$
\sigma / \sigma^{\text {bulk }}=\frac{c_{\mathrm{K}^{+}}+c_{\mathrm{Cl}^{-}}}{c_{\mathrm{K}^{+}}^{\text {bulk }}+c_{\mathrm{Cl}^{-}}^{\text {bulk }}}=\frac{c_{\mathrm{K}^{+}}+c_{\mathrm{Cl}^{-}}}{2 c_{0}}=\frac{I_{c}}{c_{0}} .
$$

where $I_{c}$ represents the local ionic strength. The normalized ionic conductivity reflects the changes of local ion concentrations in comparison with the bulk values. Current rectification can be quantitatively described by defining a rectification factor as

$$
r(\Delta \phi)=\left|\frac{I(-\Delta \phi)}{I(\Delta \phi)}\right|
$$



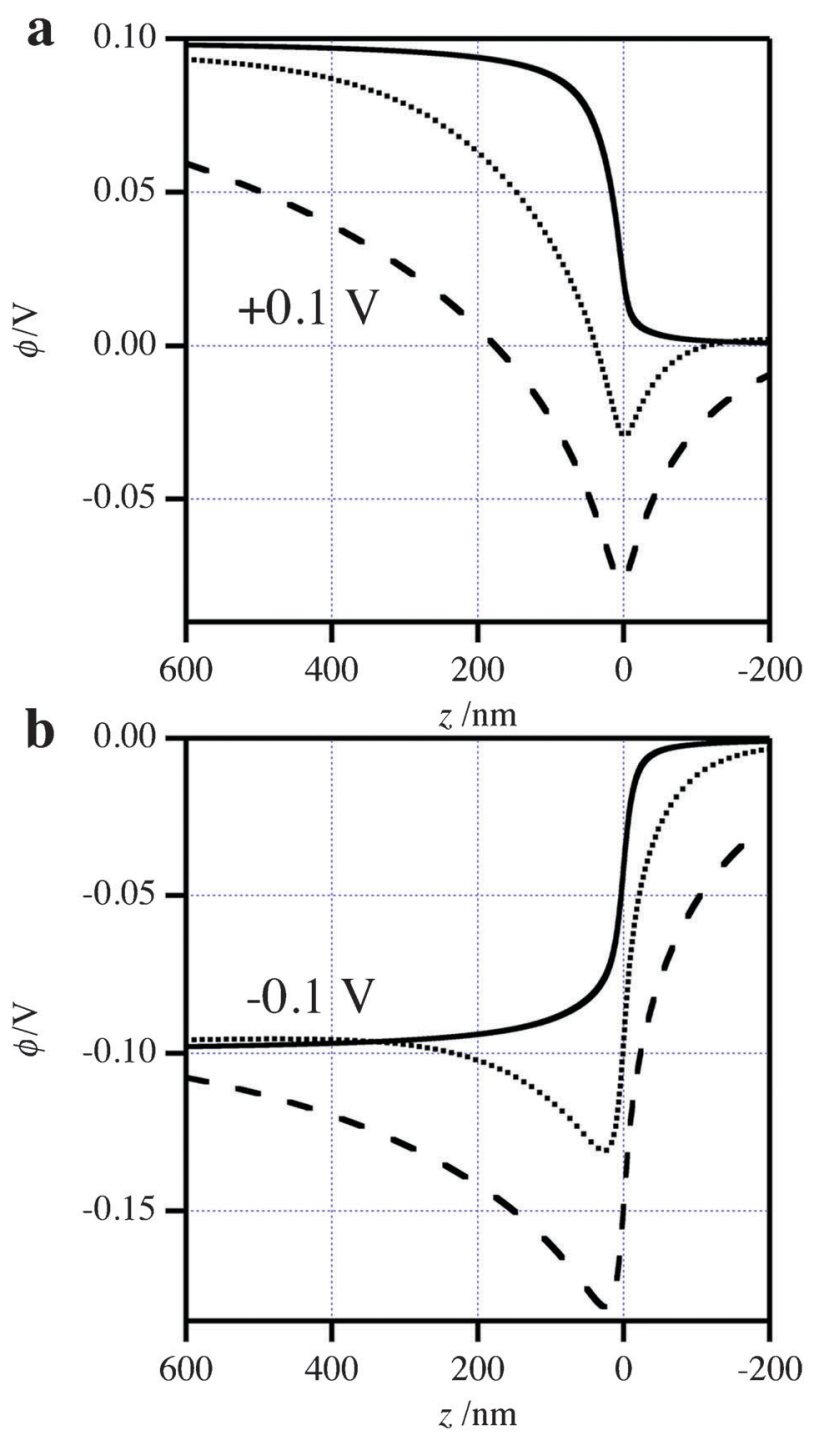

Fig. 3 Electrical potential distributions along the $z$ axis of the negatively charged nanopore for different electrolyte concentrations with a potential applied equal to (a) $0.1 \mathrm{~V}$ and (b) $-0.1 \mathrm{~V}$. Single, dotted and dashed lines correspond to $1,0.04$ and $0.005 \mathrm{mM} \mathrm{KCl}$ solutions, respectively. The pore mouth is located at $z=0$.

where $I(-\Delta \phi)$ and $I(\Delta \phi)$ are the current values for negative and positive biases, respectively.

We used finite element simulation to calculate the current flowing through the system for different biases for a conical pore having a fixed semi-angle of $30.87^{\circ}$ as a function of the electrolyte concentration, and Fig. 4 presents the variation of the rectification factor with concentration. At very low concentrations, the rectification factor is smaller than unity, then becomes larger than unity as the concentration reaches the millimolar range before tending to unity at high concentrations. This figure clearly shows that rectification can be inverted when varying the electrolyte concentration. To the best of our knowledge, this rectification inversion has not been observed before, neither experimentally nor numerically; however, previous reports show a decrease of the rectification ratio in diluted electrolytes. ${ }^{24,25}$ To discuss this variation of rectification, two limiting cases are considered.

\section{Classical rectification}

The classical rectification case observed experimentally occurs when the concentration of $\mathrm{KCl}$ is high enough, e.g. $1 \mathrm{mM}$, to keep the rectification ratio $r(\Delta \phi)$ bigger than 1 (see Fig. 4, region a and Fig. 5a). As seen in Fig. 3, the electric potential distribution is monotonous and the potential drop in the pore is equal to $79 \%$ compared to $21 \%$ outside for positive biases. The ratio of the potential drop found here to be 3.8 is quite close to that indicated by the ratio of resistance model described above and equal to 3.6.

As reported by many authors, ${ }^{22-24}$ for a positive bias, the normalized conductivity within the pore is less than unity, whereas the opposite occurs at a negative bias, the value along the $z$-axis being represented in Fig. 5 and compared to the equilibrium case. Very close to the walls an excess of positive charges is present and the local conductivity is higher than the bulk value. Actually, when examining the electroneutrality condition (see Fig. S-2 in the ESI), $\dagger$ it is clear that electroneutrality is only broken close to the wall and partially at the pore tip. Consequently, the transport number of the two ions is equal to 0.5 in the electroneutral region, but the transport number of potassium increases towards unity close to the charged wall.

The passage of a steady-state electrical current results in asymmetry of the normalized conductivity in regions that are electroneutral both inside and outside the pore. In a positive bias, the decrease of normalized conductivity, i.e. of ionic strength, occurs within the pore where the geometric factor $k_{\text {in }}$ is higher and this results in a very high resistance zone that limits the overall current. Inversely, in negative bias, the increase of normalized conductivity within the pore and a quasi-bulk conductivity outside the pore in a zone where the geometric factor $k_{\text {out }}$ is less than inside the pore results in a higher current.

This variation of conductivity in electroneutral regions stems from a Donnan effect associated to the presence of a perm-selective region at the tip, as originally suggested by Wei et al. ${ }^{13}$ To test this hypothesis, we have examined the ionic distributions in a nanopore in the absence of charge on the wall, but where a thin perm-selective plug is present at the tip.

Hereafter, we propose a simple analytical model of ionic transport in an uncharged nanopore, which presents perm-selectivity at the tip (see Fig. 1b). To calculate the current flowing through such a system, one can consider the flux eqn (1) in the absence of convection for both the cation and the anion. As for the calculation of the resistance, truncated spherical mass transport is assumed within the pore and a hemispherical outside as illustrated in Fig. 1b. At steady state, at the surface of the truncated sphere defining the perm-selective plug, we have

$$
\Omega r^{2} J_{\mathrm{K}^{+}}=\frac{t_{+} I}{F}
$$

where $t_{+}$indicates the transport number of the cations in the perm-selective plug and

$$
\Omega r^{2} J_{\mathrm{Cl}^{-}}=\frac{-\left(1-t_{+}\right) I}{F}
$$




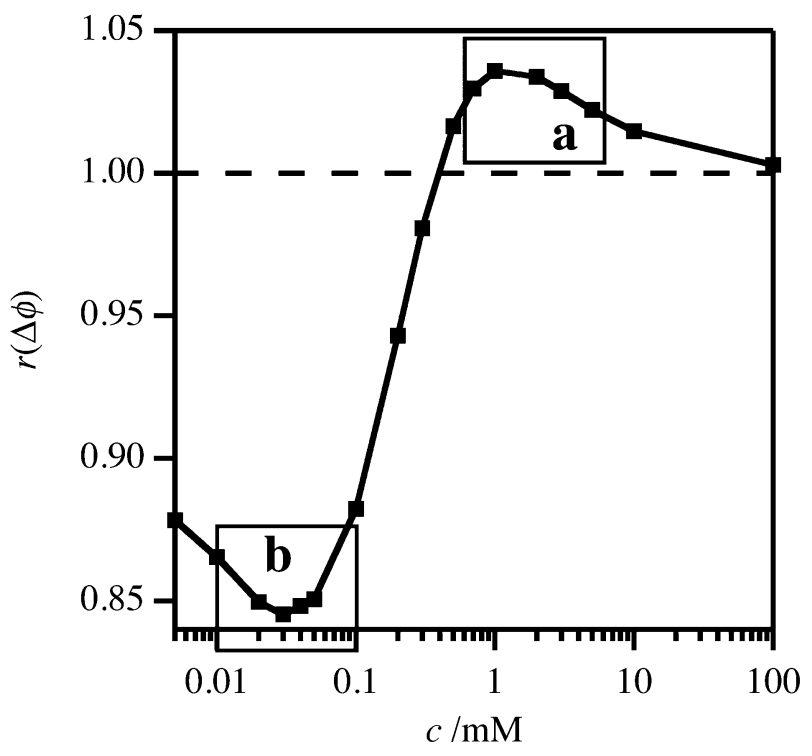

Fig. 4 Dependence of the rectification ratio $r(\Delta \phi)$ on the $\mathrm{KCl}$ concentration (given on a logarithmic scale) in a negatively charged conical nanopore. Dashed line $r(\Delta \phi)=1$ shows the absence of rectification. Regions $\mathrm{a}$ and $\mathrm{b}$ on the curve correspond to the schemes depicted in Fig. 5a and b, respectively.

describes the anion flux, which is opposite to the current direction.

In spherical coordinates, the flux eqn (1) therefore reads

$$
\frac{\partial c_{\mathrm{K}^{+}}}{\partial(1 / r)}+\frac{c_{\mathrm{K}^{+}} F}{R T} \frac{\partial \phi}{\partial(1 / r)}=\frac{t_{+} I}{\Omega F D_{\mathrm{K}^{+}}}
$$

and

$$
\frac{\partial c_{\mathrm{Cl}^{-}}}{\partial(1 / r)} \frac{c_{\mathrm{Cl}^{-}} F}{R T} \frac{\partial \phi}{\partial(1 / r)}=\frac{-\left(1-t_{+}\right) I}{\Omega F D_{\mathrm{Cl}^{-}}}
$$

By using the electroneutrality condition in solution (no charged wall, $\left.c=c_{\mathrm{K}^{+}}=c_{\mathrm{Cl}^{-}}\right)$and the equality of the diffusion coefficient of $\mathrm{K}^{+}$and $\mathrm{Cl}^{-}\left(D=D_{\mathrm{K}^{+}}=D_{\mathrm{Cl}^{-}}\right)$, we obtain the simple differential equation

$$
\frac{\partial c}{\partial(1 / r)}=\frac{I\left(2 t_{+}-1\right)}{2 \Omega F D}
$$

To link the concentration $c(r)$ to the potential drop, we have

$$
\frac{\partial c}{\partial(1 / r)}=\left(2 t_{+}-1\right) \frac{c F}{R T} \frac{\partial \phi}{\partial(1 / r)}
$$

The integration of eqn (16) inside the pore from $r_{\mathrm{p}}$ to the bulk (i.e. $r \rightarrow \infty$ ), taking into account the direction of the current, gives

$$
c_{0}-c\left(r_{\mathrm{p}}\right)=\frac{\left(2 t_{+}-1\right)}{4 \pi(1-\cos \alpha) F D r_{\mathrm{p}}}
$$

The potential drop within the pore is given by the integration of eqn (17) and reads

$$
\Delta \phi=-\frac{R T}{\left(2 t_{+}-1\right) F} \ln \left(1-\frac{I\left(2 t_{+}-1\right)}{4 \pi(1-\cos \alpha) F D c_{0} r_{\mathrm{p}}}\right)
$$

The resistance within the pore is then calculated by Ohm's law

$$
R_{\text {in }}=-\frac{R T}{\left(2 t_{+}-1\right)} \ln \left(1-\frac{I\left(2 t_{+}-1\right)}{4 \pi(1-\cos \alpha) F D c_{0} r_{\mathrm{p}}}\right)
$$

where $r_{\mathrm{p}}$ is the radius at the solution-perm-selective plug interface. Similarly, the resistance outside the pore where hemispherical mass-transport occurs is given by

$$
R_{\text {out }}=\frac{R T}{\left(2 t_{+}-1\right) I F} \ln \left(1+\frac{I\left(2 t_{+}-1\right)}{4 \pi F D c_{0} r_{\text {tip }}}\right)
$$

A permselective plug can be assumed as the zone between two truncated spheres with radii $r_{\text {tip }} \cot \alpha$ and $r_{\text {p }}$ centered at point $\mathrm{F}$. The resistance of a plug therefore is

$$
R_{\text {plug }}=\frac{\rho}{\Omega} \int_{r_{\text {tip }} \cot \alpha}^{r_{\mathrm{p}}} \frac{d r}{r^{2}}=\frac{\rho}{\Omega}\left(\frac{\tan \alpha}{r_{\text {tip }}}-\frac{1}{r_{\mathrm{p}}}\right)
$$

where $\rho$ can be approximated as the bulk value

$$
\rho=\frac{1}{\sigma_{\text {bulk }}}=\frac{R T}{2 F^{2} D c_{0}}
$$

All in all, the total resistance of the system is given by

$$
\begin{aligned}
R_{\text {tot }}= & R_{\text {in }}+R_{\text {out }}+R_{\text {plug }}=\frac{R T}{\left(2 t_{+}-1\right) I F} \\
& {\left[\ln \left(1+\frac{I\left(2 t_{+}-1\right)}{4 \pi F D c_{0} r_{\text {tip }}}\right)-\ln \left(1-\frac{I\left(2 t_{+}-1\right)}{4 \pi(1-\cos \alpha) F D c_{0} r_{\mathrm{p}}}\right)\right] } \\
& +\frac{R T}{4 \pi F^{2} D(1-\cos \alpha) c_{0}}\left(\frac{\tan \alpha}{r_{\text {tip }}}-\frac{1}{r_{\mathrm{p}}}\right)
\end{aligned}
$$

This simple analytical approximation of ionic transport through a conical pore having perm-selective properties at the tip describes the current-voltage behavior of conical nanopores with different selectivity to cations and different size of a perm-selective zone. The variation of ionic conductivity in accordance with the polarity of bias is also predicted by the model and can be obtained from integration of eqn (17) inside and outside the pore.

The model predicts an increase of the rectification ratio with increasing perm-selectivity at the tip, i.e. with the increase of cation transport number $t_{+}$. Fig. 6a illustrates the variation of the rectification factor with an increase of selectivity to cations according to eqn (24). When the pore reaches an ideal permselective state (i.e. $t_{+}=1$ ) the rectification factor tends to a maximal value of 3.20. Numerical simulations of a thin $(0.5 \mathrm{~nm})$ perm-selective plug presented at the tip give a rectification factor $r(\Delta \phi)$ equal to 2.14 . The difference observed between the analytical approximation and numerical simulations stems from a different geometrical model of a perm-selective region.

In conclusion, a nanopore with a charged wall behaves as a neutral nanopore with a perm-selective plug at the tip, with similar variation of conductivity inside and outside the pore.

The conical shape of the pore leads to the fact that masstransport inside the pore is less than outside resulting in an accumulation or depletion of cations within the tip according to the bias polarity. Consequently, a change of ionic conductivity inside and outside the pore occurs, resulting in ion 


\section{a $1 \mathrm{mM}$ Classical rectification}
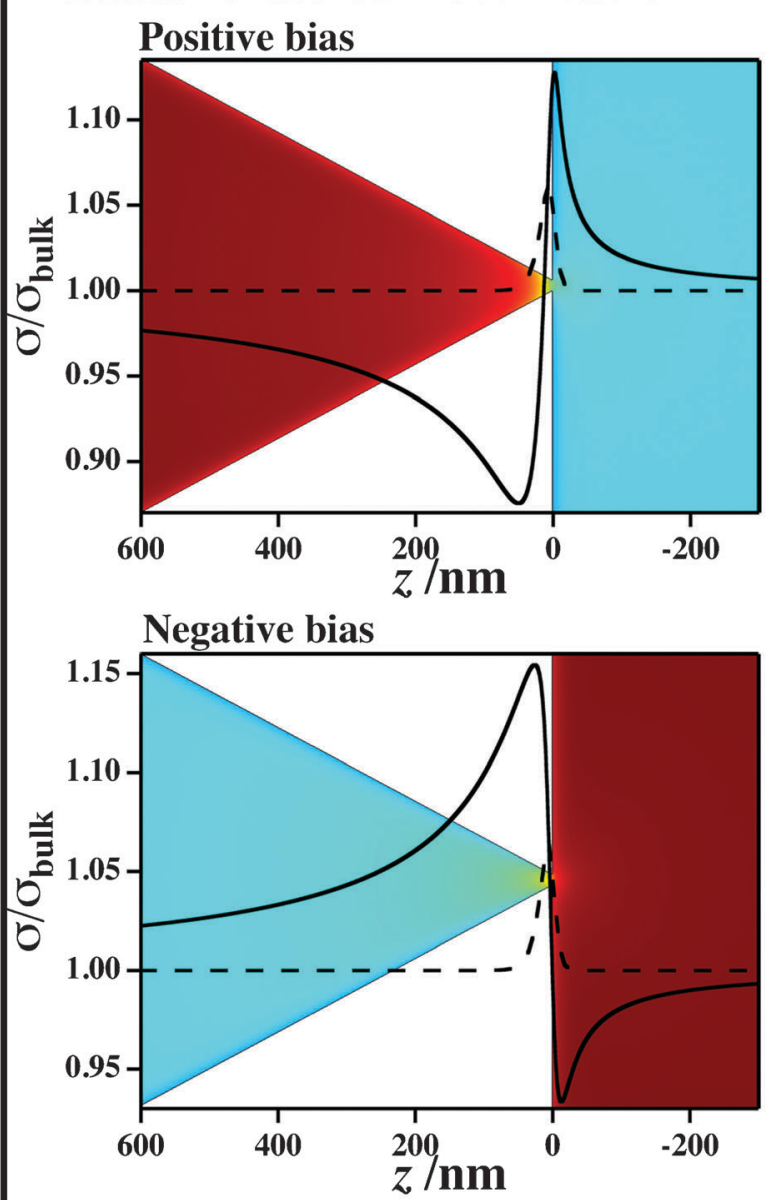

b $0.04 \mathrm{mM}$ Inverted rectification

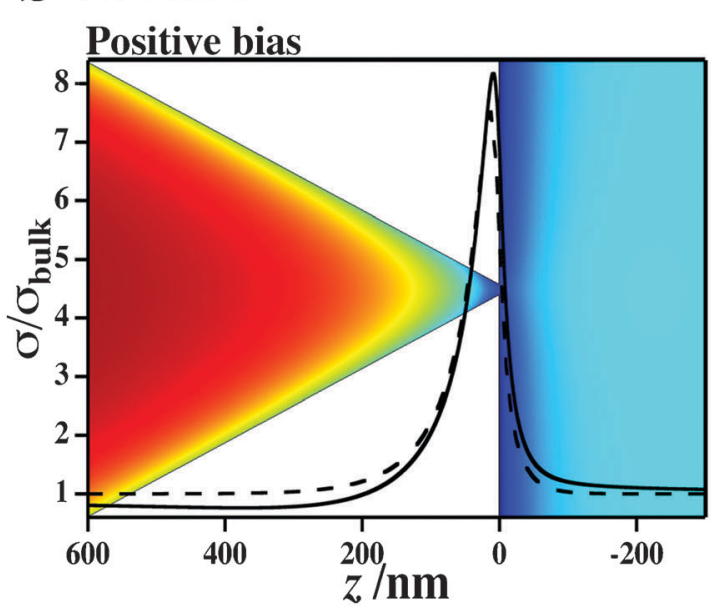
$\Delta \phi / \mathrm{V}$

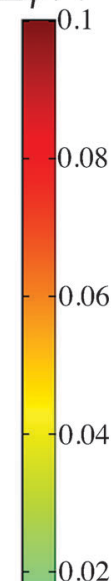

Negative bias

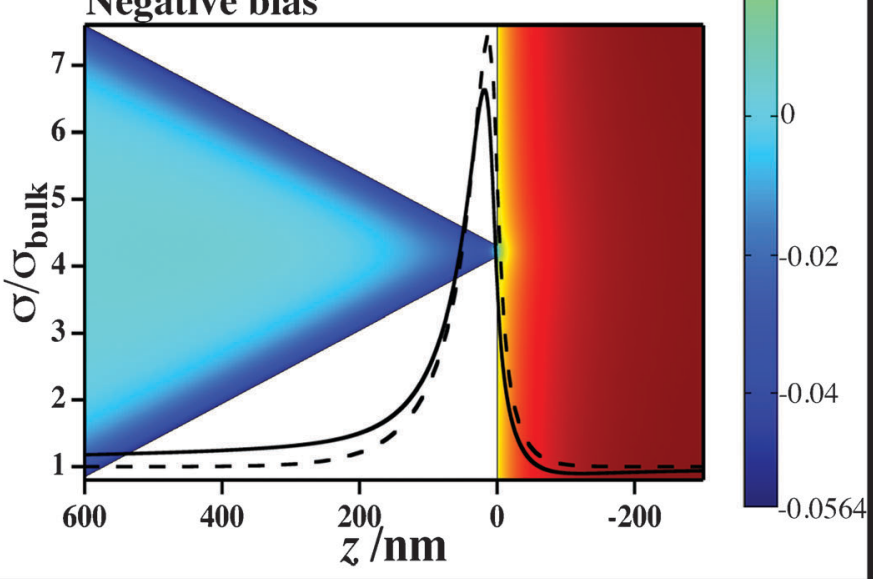

Fig. 5 Relative ionic conductivity distributions along the $z$ axis and electric potential isovalues at the tip of the charged nanopore for positive and negative biases of $0.1 \mathrm{~V}$ (solid lines) and in a static case (i.e. no bias applied, dashed lines) in (a) $1 \mathrm{mM}$ and (b) $0.04 \mathrm{mM} \mathrm{KCl}$ solutions.

current rectification. Taking into account the formation of a perm-selective zone at the tip and the expansion of the GouyChapman layer in diluted electrolytes, one can consider the growth of the rectification factor in diluted solutions as the increase of the selectivity of the pore to the counter-ions of EDL.

\section{Inverted rectification}

As shown in Fig. 4, a gradual decrease of $\mathrm{KCl}$ concentration from 100 to $1 \mathrm{mM}$ results in an increase of the rectification degree, which can be attributed to the changes of permselectivity of the pore. In more diluted solutions, $r(\Delta \phi)$ decreases as observed in previous experiments ${ }^{25}$ and predicted by theoretical analyses. ${ }^{24}$ Upon further dilution the present study predicts an inversion of the rectification effect where the current becomes higher at positive rather than negative biases. To illustrate this phenomenon, we chose a concentration of $0.04 \mathrm{mM}$ (see Fig. 4, region b and Fig. 5b). For such diluted solutions, the overlap of Gouy-Chapman layers extends deeper into the pore and this region is characterized by very high relative conductivity values due to cation accumulation at the very tip (Fig. 5b). According to the sign of the applied bias, the position of the relative conductivity maximum shifts slightly towards the tip (positive bias) or inside the pore (negative bias) like the position of a potential well formed near the pore opening (see Fig. 3). Consequently, in the former case, we have higher conductivity in the narrowest part of the tip yielding a higher current value, whereas in the latter, the high conductivity zone is shifted inside yielding a lower current value due to the presence of a more diluted solution at the tip. It is worth to note that conductivity inside the pore being at the high- and low-conductance states is higher than the bulk value, as shown in Fig. 5b. Such behavior in diluted electrolyte solutions has already been reported ${ }^{25,29}$ and is an experimental proof that the rectification factor decreases in this case. ${ }^{25}$

The analytical model of ionic transport described above also predicts the inversion of rectification with the growth of the diffuse layer in diluted electrolytes. The assumption is that the cation-enriched zone at the tip is only cation-permeable (ideal perm-selectivity). As was discussed before, the decrease of electrolyte concentration leads to the expansion of the region where Gouy-Chapman layers overlap. Fig. 6b illustrates the change of rectification factor in accordance with the thickness of a perm-selective zone within the pore. Numerical simulations of an uncharged nanopore which exhibits ideal permselectivity at the tip region show the same trend for the 


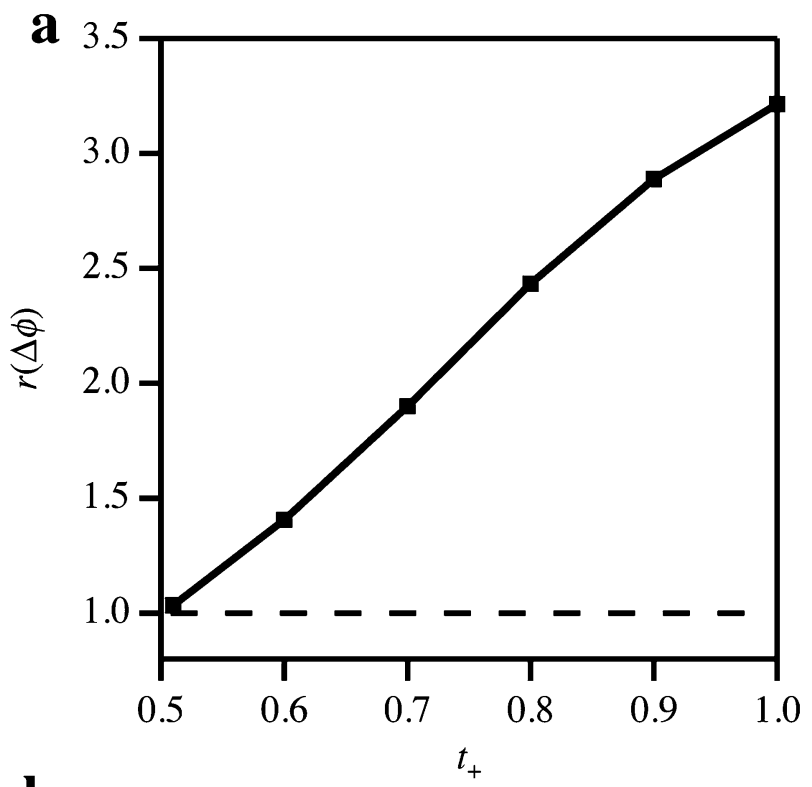

b

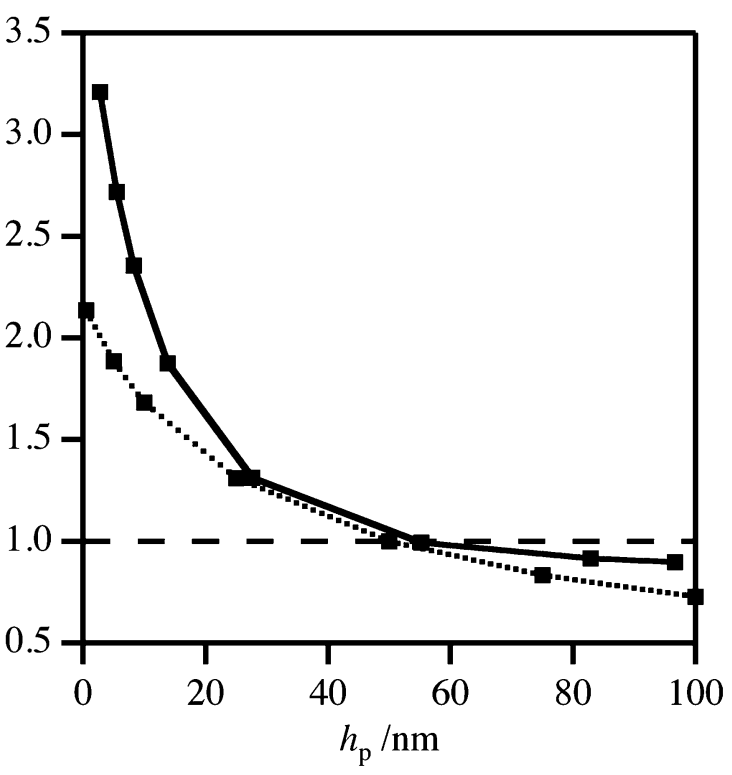

Fig. 6 The influence of (a) cation transport number in a permselective zone and (b) the thickness of a perm-selective plug on a value of rectification factor according to the analytical approximation of ionic transport in an uncharged conical nanopore (see eqn (24), solid line) and results of numerical calculations of Nernst-Planck-Poisson equations for the uncharged pore, containing perm-selective region (dotted line). The thickness of a perm-selective zone in the analytical model is assumed as $h_{\mathrm{p}}=r_{\mathrm{p}}-r_{\text {tip }} \cdot \cot \alpha$. The dashed line $r(\Delta \phi)=1$ shows the absence of rectification.

rectification ratio as predicted by the analytical model, i.e. gradual decrease of the rectification factor and even rectification inversion when a large perm-selective plug occurs inside thepore.

At even lower electrolyte concentrations (i.e. $<0.03 \mathrm{mM}$ ) the rectification factor tends to a slow increase. This observation is unexpected from the perm-selective model described above, as well as from the numerical simulations of a pore, possessing ideal perm-selective properties. Despite the high-conductivity region still appearing at the tip, considerable changes in ionic distributions occur deeper inside the pore (see Fig. S-3 in the ESI). $\dagger$ The changes of ionic conductivity far away from the tip limit the inversion of rectification. Most likely, such observation appears as a combination of both mechanisms, i.e. classical ICR (as changes of perm-selectivity of the pore) and rectification inversion (i.e. the increase of the permselective region), and the value of the rectification factor is given by the contribution from both.

\section{Influence of the pore geometry}

Rectifying properties are strongly dependent on the geometrical parameters of the pore. As was already noted, pores with smaller tip diameters present higher rectification values. ${ }^{25}$ Additionally, rectification is also dependent on the shape of the pore. ${ }^{26}$ In the present study only conical nanopores were under consideration.

In comparison with other geometrical parameters, the pore length has minor influence on the rectification ratio value, at least for pores of $1-10 \mu \mathrm{m}$ length (the results are not shown). The latter is most likely due to the fact that the rectification phenomenon occurs mainly because of the aforementioned tip effects. Another parameter that describes the asymmetry of the pore is the cone semi-angle (marked as $\alpha$ in Fig. 1). Interestingly, the influence of this angle on the rectification ratio reaches a maximum that depends on the electrolyte concentration. As shown in Fig. 7a, the inversion of the rectification direction is possible only for pores with large half-cone angles. Moreover, the change the rectification properties (i.e. to inversion of ICR direction) requires larger pore angles as higher electrolyte concentrations are employed. The reason for such behavior could be drawn from ICR mechanisms.

In case of classical rectification, decrease of a pore semiangle should result in a higher resistance of the pore. Thereby, mass-transport rate within the pore decreases and limits the ionic current, while resistance outside is not subjected to considerable changes. The result is the more effective depletion and enrichment of salt concentration observed for positive and negative biases, respectively, and higher difference in conductivities occurs. This result is predicted by the analytical model of a perm-selective pore and can be illustrated as shown in Fig. 7b. Pores that possess low perm-selectivity at the tip (i.e. at high electrolyte concentrations) exhibit weak rectifying properties in a wide range of $\alpha$. The increment of a cation selectivity leads to the increase of the rectification ratio, especially for the pores with small semi-angles values. In other words, the decrease of electrolyte concentration would result in a stronger rectification reaching a maximum at small $\alpha$.

However, further dilution causes growth of a perm-selective zone which reduces the rectification factor and leads even to inversion of the rectification direction. This change in the rectifying properties is facilitated in a pore with large halfcone angles, which shows in good agreement between the model of a perm-selective pore and numerical simulations. The rise or drop of the half-cone angle leads to a more or less effective concentration or dilution of the cation-enriched zone at the tip. The bigger angles ease the dilution of the highconductivity region at the tip, since the cone volume extends 

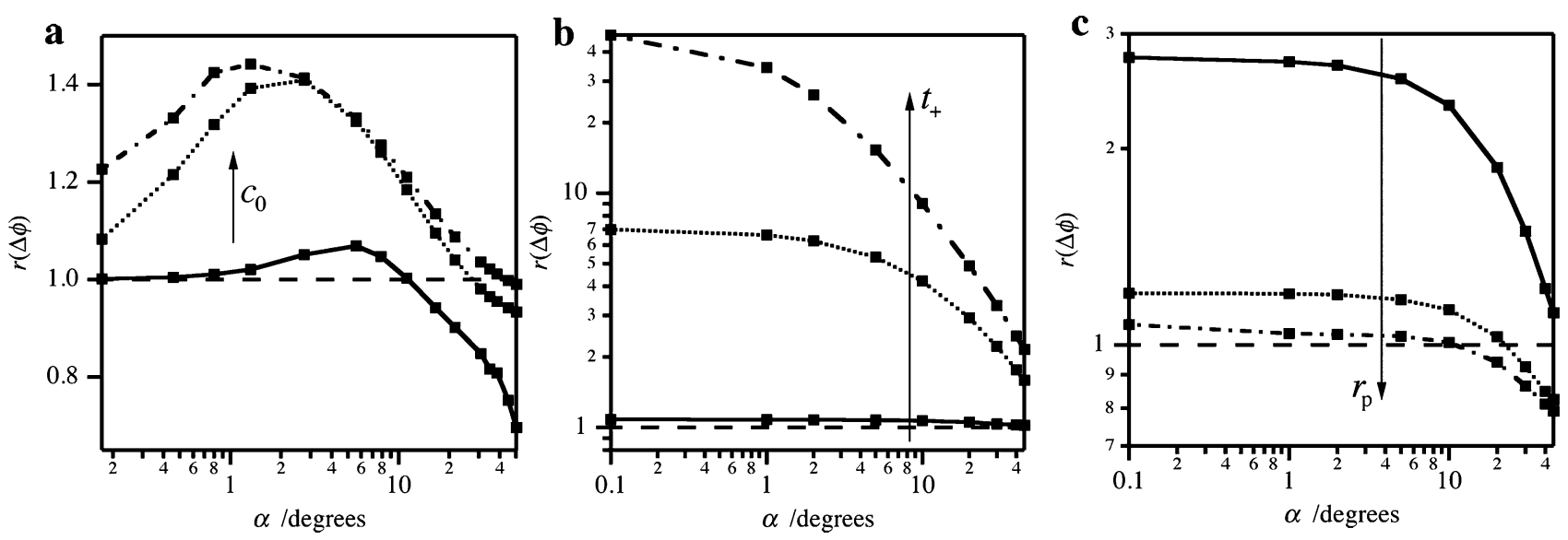

Fig. 7 Effect of the pore half-cone angle on the rectification ratio at different electrolyte concentrations. (a) The values of rectification factor according to numerical simulations of ICR in charged conical nanopores. The solid, dotted and dash-dotted curves correspond to $0.04,0.3$ and $1 \mathrm{mM} \mathrm{KCl}$ solutions, respectively. The arrow indicates increasing electrolyte concentration. (b) Rectification ratio values in accordance with analytical approximation of ICR in pores with different perm-selectivity at the tip (see eqn (24)). The solid, dotted and dash-dotted lines correspond to the values of cation transport number equal to $0.51,0.75$ and 1 . The size of the perm-selective plug is limited by $r_{\text {tip }}=10 \mathrm{~nm}$ outside and $r_{\text {tip }} \cdot \sin ^{-1} \alpha$ inside the pore. (c) Analytical approximation of ICR in pores containing an ideal perm-selective plug $\left(t_{+}=1\right)$ of different size: 2,5 and $10 r_{\text {tip }} \cdot \sin ^{-1} \alpha$ for solid, dotted and dash-dotted lines, respectively. The dashed line $r(\Delta \phi)=1$ shows absence of rectification. The rectification factor (on b) and (c) and angle values are presented on a logarithmic scale.

intensively from the cone vertex to the cone base. Consequently, the ion current value amplifies for positive biases and descends for negative ones, and inversion of the rectification direction occurs. The predictions of the analytical model shown in Fig. 7c indicate the gain of rectification inversion with higher semiangle values and larger size of a perm-selective plug.

Even so, it was shown before that cylindrical nanotubes in symmetric electrolyte conditions with homogenous charged walls exhibit a linear current-voltage characteristic, ${ }^{30}$ since the pore asymmetry is lost. Thereby, on the one hand the decrease of the cone semi-angle is manifested in the increase of the rectification ratio (as shown in Fig. $7 b$ and c), and on other hand, rectification disappears (i.e. $r(\Delta \phi)=1$ ) when the pore reaches a tube-like shape. The combination of the effects that increase and decrease the rectification ratio with the change of the angle leads to the existence of a maximum on the curves in Fig. 7a, which is not predicted by the analytical model. The latter result appears, as changes in the Gouy-Chapman layer by the pore geometry variations (i.e. half-cone angle) are not considered in the analytical model. Interestingly, the investigation of rectification in conical nanofluidic funnels show a similar trend, i.e. the increase of current at negative potentials as compared to positive ones with the change of the angle from 20 to $5^{\circ}$, and the loss of rectification in straight funnels. ${ }^{29}$

\section{Influence of applied voltage}

It is important to notice that the main electric field (i.e. $\Delta \phi \cdot l^{-1}$, where $\Delta \phi$ and $l$ denotes bias and the distance between electrodes, respectively) has almost no influence on the rectification degree, since only the values of the electric field near the large and small openings of the pore are important. The latter is explained as the electrodes position with respect to each other and the distance between cathode and anode is less important than the applied potential difference. In other words, the main influence on the electrical properties of the system has the potential drop, which occurs primarily inside the pore.

The amplification of the rectification degree by the value of the applied voltage has been shown experimentally ${ }^{17}$ and proven theoretically. ${ }^{24,26,31,32}$ Even so, it is worth to discuss the influence of a potential bias on the basis of the proposed mechanism for classical and inverted rectification.

As shown in Fig. 8, the amplification of the rectifying properties of the pore by the value of the applied bias is different for various pore geometries and electrolyte concentrations. The rectification degree in pores with small $\alpha$ is preferably amplified in case of high $\mathrm{KCl}$ concentrations, while stronger inversion of rectification is observed for diluted electrolytes within the pores with large half-cone degrees. Even so, the main trend of the rectifying level enhancement remains for pores of different geometries.

The rectification of the ion current in diluted $\mathrm{KCl}$ solutions (Fig. $8 \mathrm{a}$ and $\mathrm{b}$, dotted lines) can be discussed with the use of a scheme shown in Fig. 5b. The mechanism depicted in the scheme is still valid for different bias values and the effect of the pore angle has already been discussed. The increase of the applied voltage results in a gradual decrease of the rectification ratio for the pores with different values of half-cone angles $\left(\alpha=30.87^{\circ}\right.$ and $\alpha=11.20^{\circ}$ ). The higher bias values produce a higher electric field within the pore. Consequently, the high-conductivity zone shifts more towards the pore mouth $(\Delta \phi>0$, positive voltage) or more backwards $(\Delta \phi<0$, negative voltage). As a result, the concentration and dilution of the region enriched by $\mathrm{K}^{+}$occur more effectively with the higher value of the applied bias and rectification ratio descends. As was shown in the previous section, the pores with bigger half-cone angles demonstrate stronger inversion of rectification.

ICR in more concentrated electrolyte solutions (Fig. 8, solid lines) is governed mainly by the mechanism shown in a scheme 

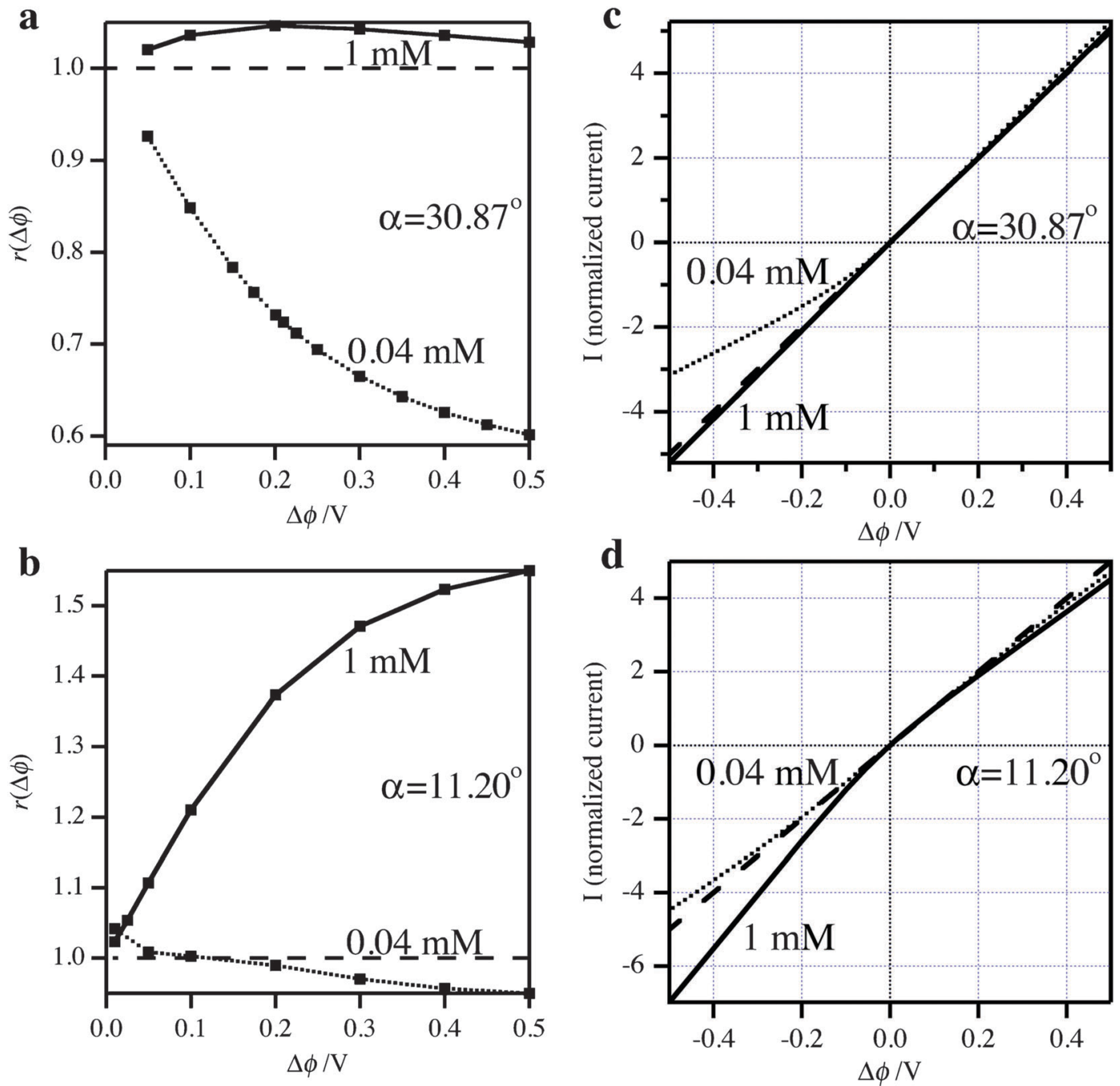

Fig. 8 Dependence of the rectification ratio on the value of applied bias for the pores with different half-cone angles $\left(30.87\right.$ and $\left.11.20^{\circ}\right) .(a),(b)$ Numerical simulations of ICR in charged nanopores. The solid and dotted lines represent curves for 1 and $0.04 \mathrm{mM} \mathrm{KCl}$, respectively. The dashed line $r(\Delta \phi)=1$ shows absence of rectification. (c), (d) Corresponding current-voltage characteristics of the pores with different semi-angles equal to (c) $30.87^{\circ}$ and (d) $11.20^{\circ}$. The current is normalized to the anodic current value at $+0.1 \mathrm{~V}$.

in Fig. 5a. The increase of the voltage in such cases leads to the increase of the electric field, which results in a more effective depletion (positive bias) or accumulation (negative bias) of salt concentration within the pore. The nanopores with lower half-cone angles rectify the ion current more effectively in this case, as discussed.

The model of a perm-selective pore described above, predicts similar trends for ICR dependence on the value of the potential bias (results not shown). The increase of a perm-selective region thickness, resulting from EDL expansion, leads to the decrease of the rectification ratio and even inversion of the ICR direction. However, the model doesn't predict the rectification inversion due to the value of applied bias, as shown in Fig. 8b.

The plateau of limiting current is not observed on currentvoltage curves shown in Fig. 8c and d. As was discussed, pores with different geometries exhibit different types of current-voltage characteristics, depending on the ionic strength conditions. The analytical approximation predicts similar diode-like behavior for the perm-selective pores (see Fig. S-4 in the ESI) $\dagger$

\section{Conclusions}

The work presented here describes the phenomenon of ion current rectification in negatively charged (e.g. glass or silica) nanopores with the emphasis on the mechanisms, which causes diode-like behavior of such systems. The results of the numerical resolution of the Nernst-Planck-Poisson equations show the existence of unusual rectification behavior, i.e. rectification inversion in diluted electrolytes, which was not observed before neither in theoretical, nor in experimental studies. In the present manuscript, we confirm the hypothesis that ICR arises due to the existence of a perm-selective region within the pore, which can be considered as a consequence of electrostatic interactions between charged walls and ionic species in an electrolyte solution. We also develop analytical and numerical models of ionic transport that consider aforementioned processes at the tip of the nanopore and demonstrate the origin of classical and inverted rectification as a consequence of permselectivity of the pore opening. In addition, the influence of the 
pore geometry and applied potential bias is investigated and discussed in terms of the proposed model of a permselective pore.

\section{Acknowledgements}

One of us (H.H.G.) is grateful to the Ministry of Education of China and Peking University for a visiting professor fellowship. This work was supported by SNCF grant no 20735001, 20628506 and by SNF grant Grant no 20PA21_121570/1

"High throughput SECM imaging".

\section{References}

1 B. Hille, Ionic Channels of Excitable Membranes, Sinauer Association, Sunderland, MA, 3rd edn, 1992.

2 J. J. Kasianowicz, E. Brandin, D. Branton and D. W. Deamer, Proc. Natl. Acad. Sci. U. S. A., 1996, 93, 13770-13773.

3 M. Akeson, D. Branton, J. J. Kasianowicz, E. Brandin and D. W. Deamer, Biophys. J., 1999, 77, 3227-3233.

4 A. Bruckbauer, L. M. Ying, A. M. Rothery, D. J. Zhou, A. I. Shevchuk, C. Abell, Y. E. Korchev and D. Klenerman, J. Am. Chem. Soc., 2002, 124, 8810-8811.

5 C. C. Harrell, Y. Choi, L. P. Horne, L. A. Baker, Z. S. Siwy and C. R. Martin, Langmuir, 2006, 22, 10837-10843.

6 Y. Fu, H. Tokuhisa and L. A. Baker, Chem. Commun., 2009, $4877-4879$.

7 A. Holtzel and U. Tallarek, J. Sep. Sci., 2007, 30, 1398-1419.

8 L. M. Ying, Biochem. Soc. Trans., 2009, 37, 702-706.

9 D. Sanchez, N. Johnson, C. Li, P. Novak, J. Rheinlaender, Y. J. Zhang, U. Anand, P. Anand, J. Gorelik, G. I. Frolenkov, C. Benham, M. Lab, V. P. Ostanin, T. E. Schaffer, D. Klenerman and Y. E. Korchev, Biophys. J., 2008, 95, 3017-3027.

10 P. K. Hansma, B. Drake, O. Marti, S. A. C. Gould and C. B. Prater, Science, 1989, 243, 641-643.
11 Y. E. Korchev, M. Milovanovic, C. L. Bashford, D. C. Bennett, E. V. Sviderskaya, I. Vodyanoy and M. J. Lab, J. Microsc., 1997, 188, 17-23.

12 Q. Li, S. B. Xie, Z. W. Liang, X. Meng, S. J. Liu, H. H. Girault and Y. H. Shao, Angew. Chem., Int. Ed., 2009, 48, 8010-8013.

13 C. Wei, A. J. Bard and S. W. Feldberg, Anal. Chem., 1997, 69, 4627-4633.

14 Z. S. Siwy, Adv. Funct. Mater., 2006, 16, 735-746.

15 L. Wang, W. Guo, Y. B. Xie, X. W. Wang, J. M. Xue and Y. G. Wang, Radiat. Meas., 2009, 44, 1119-1122.

16 R. X. Yan, W. J. Liang, R. Fan and P. D. Yang, Nano Lett., 2009, 9, 3820-3825.

17 L. J. Cheng and L. J. Guo, Chem. Soc. Rev., 2010, 39, 923-938.

18 Z. Siwy, Y. Gu, H. A. Spohr, D. Baur, A. Wolf-Reber, R. Spohr, P. Apel and Y. E. Korchev, Europhys. Lett., 2002, 60, 349-355.

19 C. C. Harrell, P. Kohli, Z. Siwy and C. R. Martin, J. Am. Chem. Soc., 2004, 126, 15646-15647.

20 Z. Siwy, E. Heins, C. C. Harrell, P. Kohli and C. R. Martin, J. Am. Chem. Soc., 2004, 126, 10850-10851.

21 D. Woermann, Nucl. Instrum. Methods Phys. Res., Sect. B, 2002, 194, 458-462.

22 D. Woermann, Phys. Chem. Chem. Phys., 2003, 5, 1853-1858.

23 J. Cervera, B. Schiedt and P. Ramirez, Europhys. Lett., 2005, 71, 35- 41 .

24 H. S. White and A. Bund, Langmuir, 2008, 24, 2212-2218.

25 M. L. Kovarik, K. M. Zhou and S. C. Jacobson, J. Phys. Chem. B, 2009, 113, 15960-15966.

26 P. Ramirez, P. Y. Apel, J. Cervera and S. Mafe, Nanotechnology, 2008, 19, 315707

27 D. Woermann, Cell. Mol. Biol. Lett., 2001, 6, 53-57.

28 D. G. G. S. H. Behrens, J. Chem. Phys., 2001, 115, 6716-6721.

29 J. M. Perry, K. M. Zhou, Z. D. Harms and S. C. Jacobson, ACS Nano, 2010, 4, 3897-3902.

30 Z. S. Siwy and C. R. Martin, Lecture Notes in Physics, 2007, 711, 349-365.

31 D. Constantin and Z. S. Siwy, Phys. Rev. E: Stat., Nonlinear, Soft Matter Phys., 2007, 76, 041202.

32 J. Cervera, B. Schiedt, R. Neumann, S. Mafe and P. Ramirez, J. Chem. Phys., 2006, 124, 104706. 